\title{
Novel Superconducting Joints for Persistent Mode Magnet Applications
}

\author{
Tayebeh Mousavi ${ }^{1}$, William Darby ${ }^{1}$, Canan Aksoy $^{2}$, Timothy Davies ${ }^{1}$, Greg Brittles ${ }^{1}$, Chris \\ Grovenor $^{1}$, Susannah Speller ${ }^{1}$ \\ ${ }^{1}$ Materials Department, Oxford University, OX1 3PH, Oxford, UK. \\ ${ }^{2}$ Electronic and Communication Department, Faculty of Technology, Karadeniz Technical University, 61830, \\ Trabzon, Turkey.
}

\begin{abstract}
Persistent current joints are a critical component of commercial superconducting magnets. The standard jointing method widely used in the magnet industry for technological low temperature superconducting wires such as $\mathrm{NbTi}$ and $\mathrm{Nb}_{3} \mathrm{Sn}$ wires uses a superconducting solder (e.g. $\mathrm{PbBi}$ ). In these joints the physical and superconducting properties of the solder materials inevitably play an important role in the overall performance of the joint. Key requirements for superconducting solders include low melting point to prevent degradation of the superconducting filaments during joining, good wettability of the superconducting filaments, suitable liquid phase viscosity, and finally adequate superconducting properties to enable sufficient supercurrent to pass through the joint under typical operating conditions (typically at $4.2 \mathrm{~K}$ in a field of $1 \mathrm{~T}$ for an MRI magnet). PbBi solder satisfies all these criteria, but restrictions on the use of lead in the magnet industry are expected in the relatively near future, so new lead-free jointing techniques need to be developed.

One approach is the development of superconducting lead-free solder materials. In our work, we have focussed on the In-Sn system and ternary systems involving In and Sn as two of the elements. Thermodynamic modelling has been used to produce ternary phase diagrams of potential alloy systems, and various formulations have been fabricated in order to explore how microstructure and phase chemistry influence the superconducting properties of the solders. Alternative approaches to fabricating lead-free joints, including spot welding and cold-pressing, have also been investigated. These methods have the potential advantage of achieving direct NbTi-NbTi joints with no intermediate, lower performance superconducting material. The spot welding method produced joints with the best superconducting performance, signifiantly better than the currently used $\mathrm{PbBi}$ solder, but the lack of reproducibility in this technique may be a problem from an industrial point of view.
\end{abstract}

\section{INTRODUCTION}

Persistent current joints between technological superconductors with operational resistances below $\sim 10^{-12} \Omega$ are essential requirements for the large magnets that are needed for devices such as magnetic resonance imaging (MRI) scanners and nuclear magnetic resonance (NMR) spectrometers. In such applications, soldering is currently employed for making persistent mode joints with extremely low resistances between $\mathrm{NbTi}$ wires using $\mathrm{Pb}$ - $\mathrm{Bi}$ alloys as the superconducting solders [1-3]. However, for environmental reasons it is crucial to develop alternative $\mathrm{Pb}$-free solders which satisfy the basic requirements for superconducting solders in this application, such as low melting temperature (lower than $500^{\circ} \mathrm{C}$ to protect the $\mathrm{NbTi}$ wires), 
appropriate thermal and mechanical properties and a high enough critical current density under typical practical operating conditions $(4.2 \mathrm{~K}$ and $1 \mathrm{~T})$.

The requirement for low melting points means that the superconducting solder systems are usually eutectic alloys in which at least the majority phase must have good superconducting properties [4-5]. Some Pb-free superconducting solders were briefly explored in the literature in the 1960s, containing at least two low melting point elements selected from $\mathrm{Sn}, \mathrm{Bi}, \mathrm{Cd}, \mathrm{In}, \mathrm{Zn}$ and $\mathrm{Sb}[5,6]$. No recent research has been done on these $\mathrm{Pb}$-free solders because $\mathrm{PbBi}$ alloys shows such excellent superconducting properties and could easily satisfy the needs of magnet manufacturers. Of the Pb-free solders, $\mathrm{Sn}$-In alloys seem to have the most promising superconducting properties according to the limited available reports [6]. We have studied this system in detail to understand the relation between superconducting properties and the microstructure to see if there are opportunities to produce alloys to compete with $\mathrm{PbBi}$ by optimising the microstructure [7,8]. It was shown that the superconducting properties of both $B$ and $\gamma$ phases improve with increasing solute concentration, and that the In-rich $B$ phase has better superconducting properties than the Sn-rich $\gamma$ phase. Moreover, the interphase boundaries are active pinning sites and so refinement of the microstructure results in an enhancement in critical current densities [7]. However, even with optimised performance, the superconducting properties of the binary $\mathrm{Sn}$-In alloys ( $\mathrm{J}_{\mathrm{C}}$ and $\mathrm{B}_{\mathrm{C} 2}$ values) are much lower than those required in industry and still need to be improved [7,9]. One approach to improve these properties can be adding a third element to binary Sn-In alloys. This study focuses on the effect of adding $\mathrm{Bi}$, Ta and $\mathrm{Sb}$ to the $\mathrm{Sn}-$ In system, and discusses how the presence of the third element alters the microstructure and superconducting properties of Sn-In solders.

\section{EXPERIMENT}

Different Sn-In-A (A: Sb, Ta, Bi) alloys (listed in Table 1) were fabricated from commercial $\mathrm{Sn}, \mathrm{In}, \mathrm{Ta}, \mathrm{Sb}$ and Bi powders (with purities better than 99.9\%) by weighing the powders and melting the mixture at temperatures up to $400^{\circ} \mathrm{C}$ on a hot-plate. The melt was either cast into a $\mathrm{Cu}$-mould or sucked into pre-heated quartz tubes to form convenient cylinders with 2 mm diameter for magnetometry measurements. The superconducting properties were investigated by measuring the magnetization using a Quantum Design SQUID magnetometer. MagnetisationTemperature curves were obtained in a range of applied magnetic fields.

The microstructure and chemistry of the samples were characterized using scanning electron microscopy (SEM) and energy-dispersive $\mathrm{x}$-ray (EDX) analysis respectively in a Jeol 5510 SEM with OI SDD detector and a Zeiss Evo SEM, both operating at 20kV accelerating voltage. The phase maps were generated by the Oxford Instruments Aztec software, and the average composition of each phase and the phase fraction were calculated from reconstructed spectra from each phase in the map.

\section{DISCUSSION}

\section{SnInSb-1: Sn-30wt\%In-5wt\%Sb}

Our first attempt to improve the superconducting properties of Sn-In solders was by the addition of $\mathrm{Sb}$. A small amount of $\mathrm{Sb}(5 \mathrm{wt} \%)$ was added to the $\mathrm{Sn}$-In solder to investigate how $\mathrm{Sb}$ reacts with the Sn-In alloys, and whether it can be dissolved into the $\beta$ and $\gamma$ phases and improve 
Table 1- The list of samples studied in this work and their compositions.

\begin{tabular}{|c|c|c|c|c|c|}
\hline Sample & Sn (wt\%) & In (wt\%) & Bi (wt \%) & Sb (wt\%) & Ta (wt\%) \\
\hline SnTa & 80 & - & - & - & 20 \\
\hline SnInTa & 31 & 59 & - & - & 10 \\
\hline SnInSb-1 & 65 & 30 & - & 5 & - \\
\hline SnInSb-2 & 35 & 50 & - & 5 & - \\
\hline SnInBi & 35 & 50 & 15 & - & \\
\hline
\end{tabular}

their superconducting properties. Figure 1 shows the elemental maps of sample SnInSb-1 along with the extracted phase map. As the $\mathrm{Sb}$ map shows, $\mathrm{Sb}$ is segregated into individual islands where the In content is also high. Apart these In-Sb rich islands, the In and $\mathrm{Sn}$ are uniformly distributed across the sample making a uniform matrix. The phase map extracted from this EDX mapping data shows two distinct phases. From the reconstructed EDX spectra, the composition and phase fraction of each phase were obtained (Figure 1e and f). The microstructure is composed of $\gamma$-SnIn (with composition $\mathrm{Sn}-24 \mathrm{wt} \% \mathrm{In}-2 \mathrm{wt} \% \mathrm{Sb}$ ) as the majority phase forming the matrix, and a small fraction of $10 \mu \mathrm{m}$ ternary InSbSn islands (approximately 4 vol\% of the whole sample) with a composition of In-36wt\% Sb-14wt\% Sn. This result shows that Sb only has a low solubility in the matrix SnIn- $\gamma$ phase.
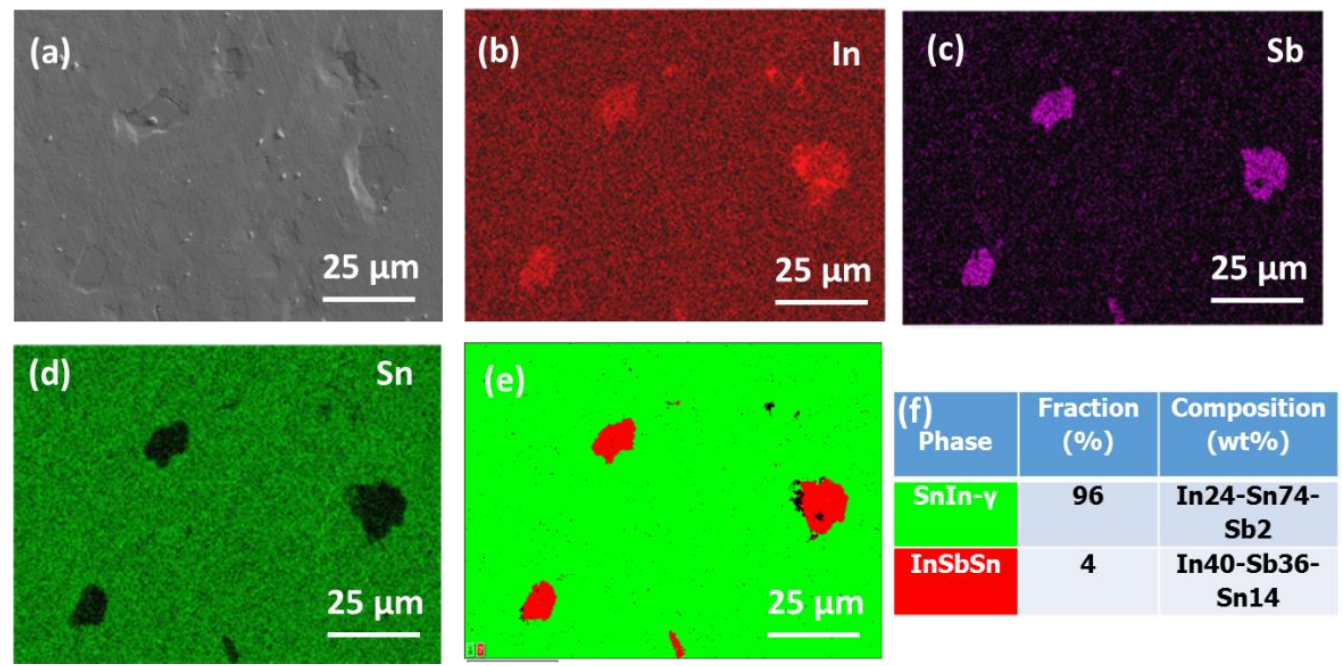

Figure 1: (a) SE image, (b-d) In, Sb and Sn maps, (e) phase map, and (f) table showing phase fraction and the composition of each phase in sample SnInSb-1.

\section{SnInSb-2: Sn-50wt\%In-5wt\%Bi}

In the first alloy, the indium reacted with $\mathrm{Sb}$ to form the islands of the ternary InSb-rich phase. This led to a reduction of In content in the matrix resulting in the formation of only the Snrich $\gamma$ phase. However, it has been shown that the In-rich $\beta$ phase has better superconducting properties than the Sn-rich $\gamma$ phase [7]. By increasing the In content in the second sample (Sn$50 \mathrm{wt} \% \mathrm{In}-5 \mathrm{wt} \% \mathrm{Sb}$ ), the aim was to form a substantial volume fraction of the In-rich $\beta$ phase, and to investigate how $\mathrm{Sb}$ reacts with this phase. Typical elemental maps of this alloy are presented in Figure 2. As can be seen, the precipitates of the ternary InSbSn phase still exist at a similar scale, chemistry and volume fraction compared to the previous sample. However, the Sn and In segregate 
at a scale of 10 microns leading to the formation of two phases in the matrix: Sn-25wt\%In (Snrich $\gamma$ phase) and In-40wt $\%$ Sn (In-rich $\beta$ phase). Both of these phases contain only a small amount of $\mathrm{Sb}$ (about 2\%) showing that $\mathrm{Sb}$ has limited solubility in the $\beta$ as well as the $\gamma$ superconducting phase.
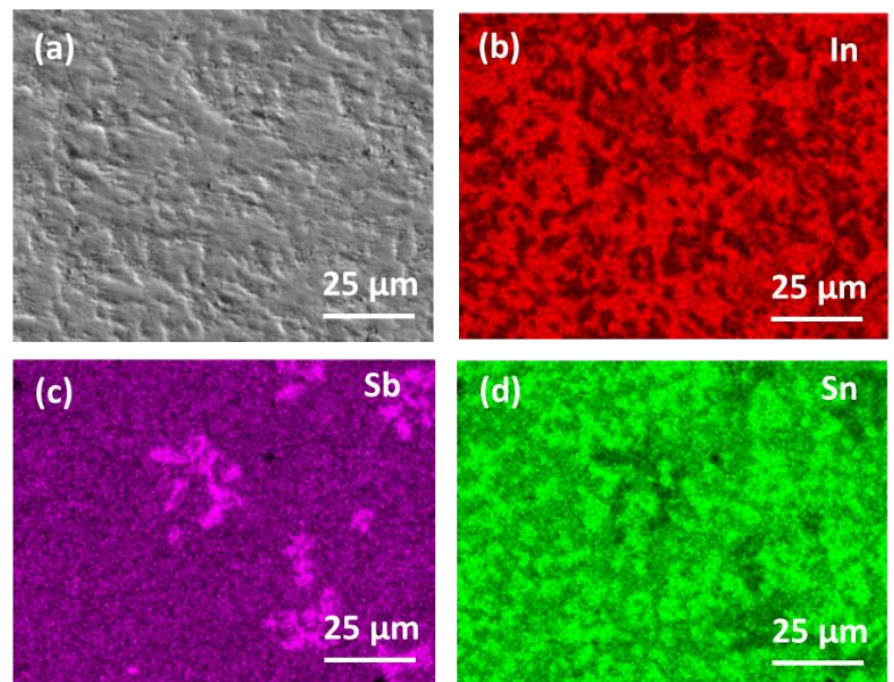

Figure 2: (a) SE image, (b-d) In, Sb and Sn maps of sample SnInSb-2.

\section{$\underline{\text { Sn-In-Ta system }}$}

It has been reported that $\mathrm{SnTa}_{3}$ has $\mathrm{T}_{\mathrm{C}}$ and $\mathrm{B}_{\mathrm{C} 2}$ values of about $8 \mathrm{~K}$ and 6080 Oe respectively [9], higher than the typical values in Sn-In alloys. If this phase is formed in a low meltingtemperature matrix (eg. Sn or SnIn), the mixture might have the advantages of both low-melting temperature and good superconducting properties, and so may perform as a good superconducting solder. To investigate this, samples of binary Sn-Ta and ternary Sn-In-Ta were made by adding Ta powder $(3-5 \mu \mathrm{m})$ into a Sn bath and Sn-65wt\% In bath respectively at about $400^{\circ} \mathrm{C}$ (the practical temperature for soldering superconducting wires). The mixtures were held for 2 hours at this temperature. Unfortunately, SEM analysis of these samples shows that no reaction occurs between the solid Ta and liquid Sn at this temperature. As can be seen in Figure 3, pure metallic Ta particles are present in the matrix with no reaction with $\mathrm{Sn}$. Similar results were found for the SnInTa sample (ie. no reaction between $\mathrm{Ta}$ and $\mathrm{In}$ ) showing that such reactions need higher temperatures which will be far from the practical temperature for soldering of superconducting wires.
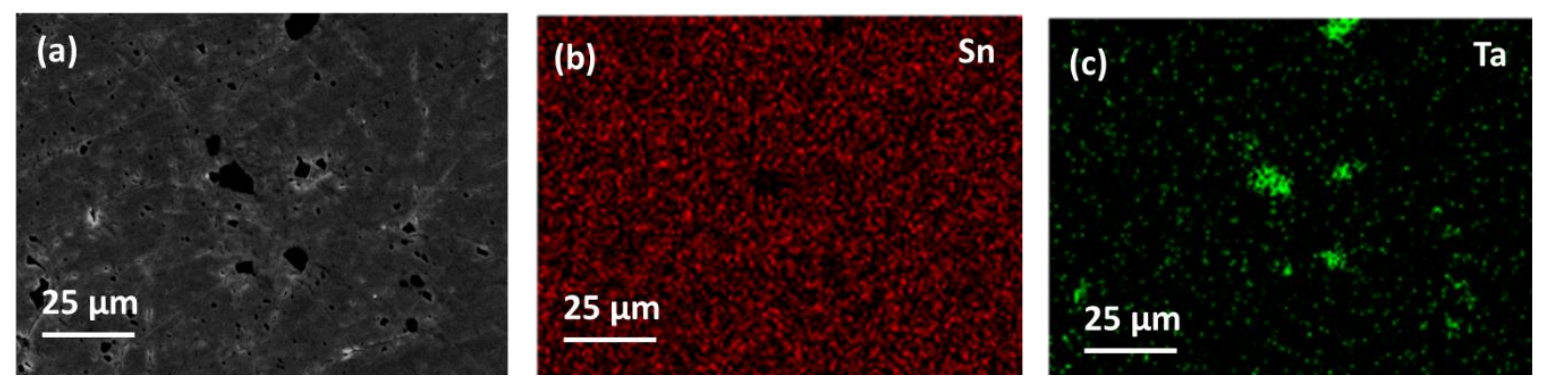

Figure 3: (a) SE image, (b) Sn map and (c) Ta map of sample Sn-Ta. 


\section{Superconducting properties}

Figure 4 shows $\mathrm{B}_{\mathrm{C} 2}$ values as a function of temperature for the samples studied in this work. For comparison, the superconducting properties of $\mathrm{PbBi}\left(\mathrm{Pb}_{60} \mathrm{Bi}_{40}\right)$, the $\mathrm{Sn}(\mathrm{In})-\gamma$ phase, $\mathrm{Sn}(\mathrm{In})-\beta$ phase and ternary SnInBi alloys studied in earlier work [7] have been also added to the graph. By comparing sample SnInSb-1, which mostly contains the $\gamma$ phase with a low concentration of dissolved Sb, with the pure $\mathrm{Sn}(\mathrm{In})-\gamma$ phase, it can be found that the presence of Sb in $\gamma$ does improve the superconducting properties, particularly the $\mathrm{T}_{\mathrm{c}}$ value which is increased from $4.4 \mathrm{~K}$ to $5.5 \mathrm{~K}$. Similarly, by comparing the SnInSb-2 sample, which is mostly composed of the $\operatorname{In}(\mathrm{Sn})-\beta$ phase with dissolved $\mathrm{Sb}$, with the pure $\operatorname{In}(\mathrm{Sn})-\beta$ phase, the dissolved Sb seems to increase both $\mathrm{T}_{\mathrm{C}}$ and $\mathrm{B}_{\mathrm{C} 2}$ values. As a result, adding $5 \mathrm{wt} \% \mathrm{Sb}$ to the $\mathrm{Sn}$-In alloys leads to a modest improvement in overall superconducting properties. Similar results have been obtained in the Sn-In-Bi system where the addition of $\mathrm{Bi}$ results in solders that show better superconducting properties than the binary Sn-In alloys [7]. According to Figure 4, alloys in the SnInBi system show higher $\mathrm{T}_{\mathrm{C}}$ and $\mathrm{B}_{\mathrm{C} 2}$ values than the SnInSb alloys tested here. However, it should be noted that the ternary $\mathrm{SnInBi}$ alloy reported in Figure 4 is the best alloy we have have found in this system in terms of superconducting properties based on a systematic study of different ternary alloys, whereas in the $\mathrm{Sn}-\mathrm{In}-\mathrm{Sb}$ system, only two different compositions both containing only $5 \mathrm{wt} \% \mathrm{Sb}$ were studied, and there might be other alloys in this system with better $\mathrm{T}_{\mathrm{C}}$ and $\mathrm{B}_{\mathrm{C} 2}$ values. It can be also noticed that the superconducting parameters of all these $\mathrm{Pb}$-free alloys are still considerably lower than those for $\mathrm{PbBi}$, indicating that we have not yet found a suitable replacement for the PbBi solder.

The SnTa sample also shows low $\mathrm{T}_{\mathrm{C}}$ and $\mathrm{B}_{\mathrm{C} 2}$ values, similar to those of $\mathrm{Sn}$ [10]. This is expected from the microstructure of this alloy which is composed of a pure Sn matrix with no dissolved Ta (Figure 3).

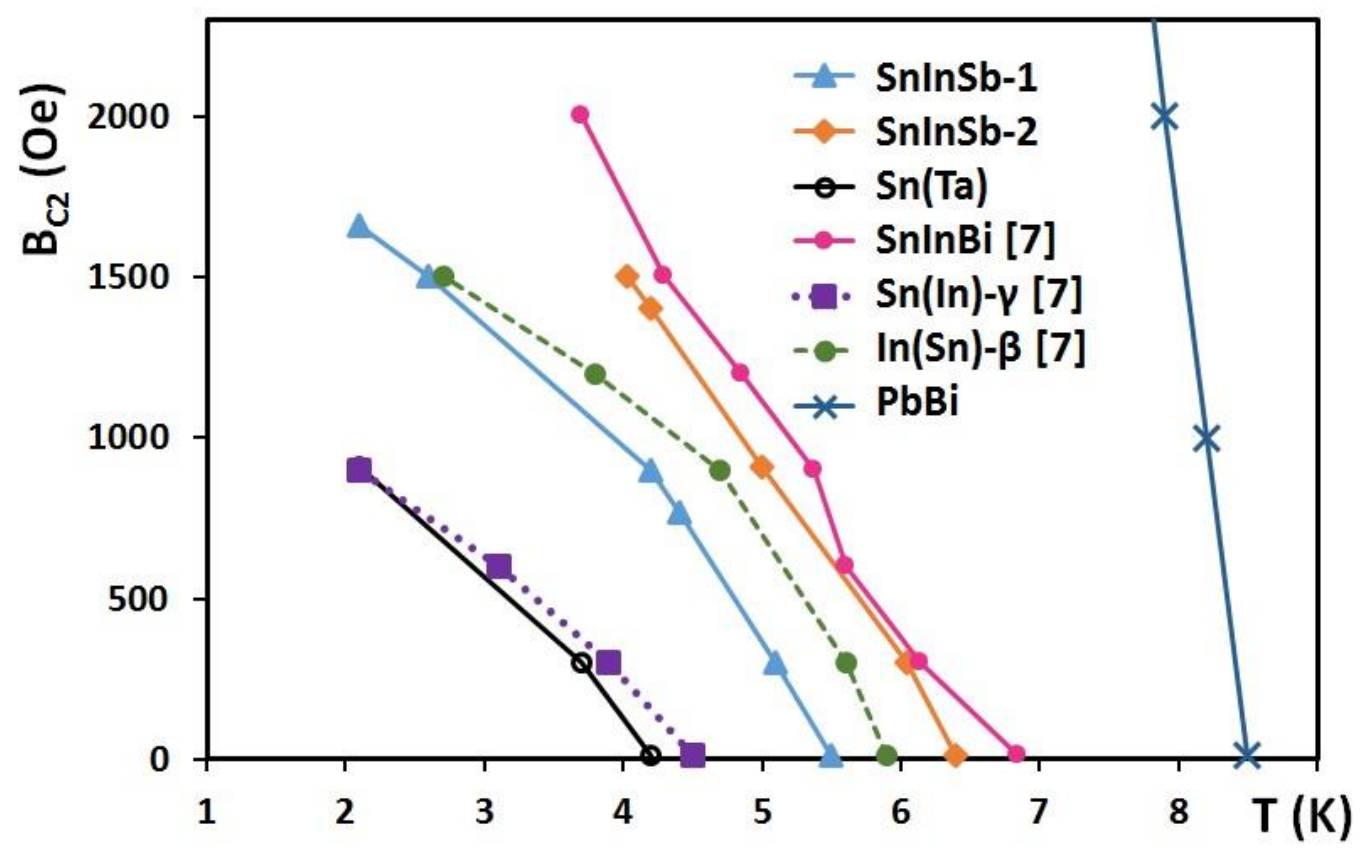

Figure 4: (a) $\mathrm{B}_{\mathrm{C} 2}$ as a function of temperature for the different solder samples studied in this work and some samples from [7]. (Each data point in the graph is the transition temperature obtained from a M/T measurement at the corresponding applied field). 


\section{CONCLUSIONS}

The superconducting properties of Sn-In binary alloys can be improved by dissolving a third element. By adding either Sb or Bi into the Sn-In phases (Sn-rich $\gamma$ and In-rich $\beta$ ), the $\mathrm{T}_{\mathrm{C}}$ and $\mathrm{B}_{\mathrm{C} 2}$ values can be increased significantly. At the temperatures we have used, Sb shows lower solubility in these phases than Bi. Up to $2-3 \mathrm{wt} \% \mathrm{Sb}$ can be dissolved into the SnIn phases at room temperature, and the excess $\mathrm{Sb}$ in the alloy will form the islands of an SbIn-rich phase. Bi has a higher solubility in both $\gamma$ and $\beta$, and has a greater influence on enhancing the superconducting parameters of these Sn-In phases. However, both of these two ternary systems have much lower $\mathrm{B}_{\mathrm{C} 2}$ and $\mathrm{T}_{\mathrm{C}}$ values than the currently used $\mathrm{PbBi}$ alloys, and cannot be considered as a good replacement for this alloy unless their properties are further improved. In the Sn-In-Ta system, no reaction occurs at the practical temperature of soldering $\left(400^{\circ} \mathrm{C}\right)$ between $\mathrm{Ta}$ and $\mathrm{Sn}$ - In, and no solubility of Ta in the Sn-In superconducting phases was observed. As a result, the microstructure is composed of a matrix of Sn-In phases with unreacted Ta particles, and $\mathrm{T}_{\mathrm{C}}$ and $\mathrm{B}_{\mathrm{C} 2}$ values show no improvement.

\section{ACKNOWLEGEMENTS}

The authors are working in the Oxfordshire Centre for Applied Superconductivity, and acknowledge the Oxfordshire Local Enterprise Partnership for financial support. Data in support of this paper will be made accessible on the Oxford Research Archive (www.ora.ox. ac.uk) doi: 10.5287/bodleian:b7GB7Xqvo.

\section{REFERENCES}

1. R.F. Thornton, U.S. Patent No. 4584547, (1986).

2. T. Fukuzaki, H. Maeda, S. Matsumoto, S. Nimori, S. Yokoyama and T. Kiyoshi, IEEE Trans. Appl. Supercond. 16, 1547-9, (2006).

3. C. A. Swenson and W.D. Markiewicz, IEEE Trans. Appl. Supercond. 9, 185-8, (1999).

4. S. A. Levy, J. Appl. Phys. 37, 3659, (1966).

5. J. E. Evetts and J.M. Wade, J. Phys. Chem. Solids. 31, 973-82, (1970).

6. G. Brittles, T. Mousavi, C. Aksoy, C. Grovenor and S. Speller, Supercond. Sci. Technol. 28, 093001, (2015).

7. T. Mousavi, C. Aksoy, C. Grovenor, S. Speller, Supercond. Sci. Technol. 29, 015012, (2016).

8. T. Mousavi, C. Aksoy, C. Grovenor, S. Speller, Trans. Appl. Supercond., doi. 10.1109/TASC.2016.2514843, (2016).

9. C. Aksoy, T. Mousavi, G. Brittles, C. Grovenor, S. Speller, Trans. Appl. Supercond., doi. 10.1109/TASC.2016.2539423, (2016).

10. Giles F. Carter and Donald E. Paul, Materials Science and Engineering, ASM International, (1991). 